\title{
Editorials
}

\section{Making technology-enabled health care work in general practice}

\section{TECHNOLOGY-ENABLED CARE SERVICES AND THE NEED FOR DIGITAL CAPABILITY} Technology-enabled care services (TECS) are gaining increasing recognition for their potential roles in underpinning delivery of care in general practice and across organisational boundaries. TECS include: video consultation/Skype/telemedicine, telehealth (information giving or interactive), apps, social media (for example, Facebook or Twitter), assistive technology/telecare, and online resources (for example, content of specific websites).' Enthusiasts expect TECS to help individual patients to live healthier lives, better manage their own health and wellbeing, and reduce demand on local services so that the majority of the population can be supported in efficient ways, leaving traditional and increasingly scarce face-to-face resources focused on those with complex health conditions.

A national report ${ }^{2}$ from Health Education England and the Royal College of Nursing highlights the need for nurses to be digitally literate. Practice nurses should facilitate, guide, and support patients along their healthcare pathways. That should enhance patients' understanding of their health and wellbeing, and empower self-care and shared care via associated modes of TECS, rather than the nurse simply being an authority figure who provides care on their own terms by 'doing things' to patients. Access to all aspects of technology with appropriate accessibility and availability will update and empower patients. This can be potentially threatening to the present nurse (or GP)-patient relationship, hence the reluctance of some clinicians to embrace this new thinking and approach in general practice.

\section{SELECTING A SUITABLE MODE OF DELIVERY}

There are many assumptions by clinicians that patients, especially older ones, are incapable of using technology. Patients are often unaware of what TECS are available in general practice. They may not use available resources to their full extent; for example, patients may go online to order repeat prescriptions, but not access their own medical records and learn more about the state of their health condition(s).

Often it is the clinicians who fail to inform patients about what technology is available, and this is generally because

\section{"Often it is the clinicians who fail to inform patients}

about what technology is available...

they have not themselves engaged with it, so, even when they do tell patients, they can be very half-hearted about the offer, and sometimes give incorrect information, such as recommending an app for a smartphone, which 'can be downloaded to your computer'.

Selecting a particular mode of delivery for a clinical consultation depends on many aspects of care. These include: the need for shared decision making and shared management between patient and clinician; availability and accessibility of TECS (in relation to NHS and patient/carer ownership); patient's/carer's preference(s); self-care and/or continuity of care recommended; established relationship, trust, and understanding between patient/ carer and practitioner; whether it is a followup or first presentation; severity and urgency of clinical management or prevention of disease or deterioration; availability of the right level of clinician (for example, GP, practice nurse, or advanced nurse practitioner); appropriate risk management; working across organisational boundaries; making the best use of technology; whether the mode of TECS or associated equipment for biometric assessment by patient is affordable and sustainable; the gaining of informed patient consent; quality, safety, and efficiency; expected outcomes being clear and reasonable; there being good quality and safety underpinning mode of TECS; competence and confidence of practitioner(s) involved; and patient's/ carer's preferred mode(s) of TECS. ${ }^{4}$

\section{The Flo telehealth sytem}

Advice relayed by technology needs to be 'user friendly' and trustworthy. Patients overwhelmingly like the use of simple Flo telehealth text messaging, for instance, for various health conditions such as hypertension, asthma, and COPD, with 95\% or more of those who replied to automated texted questions preferring remote monitoring, rather than visiting their GP surgery. ${ }^{5}$ Clinicians were supportive too in this study. Seventy-seven per cent of those offered Flo simple telehealth felt that Flo was easy to use, 58\% thought that Flo helped patients to understand their condition better, and $55 \%$ felt that the readings sent in were accurate. Only 35\% felt that Flo saved them time, $29 \%$ felt that patients had contacted the service less than before, and $16 \%$ reported a reduction in emergency hospital admissions. ${ }^{5}$

\section{Text messaging}

Text messaging has helped a number of patients to change their behaviour, and increase compliance with medication or other clinically recommended interventions. Patients with greater understanding of how to manage their condition and undertake self-care make fewer demands on health professionals. 5,6

\section{Facebook}

Facebook is a mode of communication with patients that the majority of general practices in northern Staffordshire have now adopted. ${ }^{7}$ For a recent review into local maternity services, more than 230 young mothers, young fathers, and pregnant women volunteered for the consultation within 2 weeks of the Facebook invite being sent out across 60 or so practices (with an approximate 400000 registered patient

it makes sense to get on with it and transform our conventional face-to-face delivery of care in general practice to modes of TECS that meet patients' needs and preferences as well as being more effective and productive for general practice teams ... 
population). Facebook has been trialled for different health conditions, with local health professionals inviting individual patients with a defined long-term condition to join a closed Facebook group and adding regular health messages as well as monitoring Facebook members' discussions and peer-to-peer support. $^{8}$

\section{Skype}

Clinicians in general practice and nursing home staff can use Skype for video consultations between GPs based in practice with full access to patients' medical records and the patient(s) residing in the home. GPs describe being able to resolve at least $50 \%$ of the contacts without the need for a home visit, and nursing home staff reported that they felt more confident managing patients in the home, rather than sending them to hospital, because of the access to the GP via Skype.

\section{PRACTICAL CONSIDERATIONS OF USING THE TECHNOLOGY}

There needs to be adequate $\mathrm{Wi}-\mathrm{Fi}$ in health centres so that clinicians can be confident in demonstrating technology to patients, or having a good Skype connection. Wherever a patient is, there needs to be adequate internet connection to enable the use of Skype and Facebook. However, not all apps require constant $\mathrm{Wi}-\mathrm{Fi}$ access, and text messaging relies on a phone signal. Nursing and care homes need to have good Wi-Fi coverage if they are to link to GP practices for video-consultations. So each home needs to have a convenient tablet, preferably attached to a trolley, which can be taken to the patient's bedside rather than the patient having to be transported to an office or treatment room where there may be a broadband socket. Some homes use this approach for their residents for inter-family Skype conversations already.

Patients who need to use technology for shared management of their longterm condition(s) may require trustworthy equipment for the patient/carer to assess their condition, such as a blood pressure monitor, pulse oximeter, thermometer, peak flow meter, inhaler, or blood glucose monitor, all of which need to be available either via the NHS or through personal purchase. They will need a mobile phone capable of sending text messages at the very least, but for apps, Skype, and websites a smartphone or tablet are necessary. As well as the equipment, they need the cognition and ability to use text messages, download apps, sign up to use Facebook, or access Skype or Patient Online.

\section{THE FUTURE OF FACE-TO-FACE CARE}

Health professionals in general do not like change. They have been educated and worked in a healthcare system where patients come to them for advice and treatment, and they are wary of educating patients to manage more on their own, that is, self-care and/or shared care. Establishing a culture where patients are partners in the management of their health condition/redressing of adverse lifestyle habit(s) is a move with which many clinicians are uncomfortable.

GPs, practice nurses, and healthcare assistants are at the forefront of this transition. It is important to include other members of the team, such as receptionists, who often have regular contact with patients. General practice needs to operate with a supportive frontline working environment, where there is a learning culture that encourages and supports transformation via new models of delivery of care; this is integral to the wider delivery of NHS and social care, with connecting TECS.

The extent to which remote care effectively supplements, underpins, or replaces faceto-face care remains to be seen, as there is very little research evidence. But it makes sense to get on with it and transform our conventional face-to-face delivery of care in general practice to modes of TECS that meet patients' needs and preferences as well as being more effective and productive for general practice teams - mirroring how people have converted to using technology in their everyday lives.

\section{Ruth Chambers OBE,}

GP Clinical Chair, Stoke-on-Trent CCG, Stoke-onTrent; Clinical Lead, the Long Term Conditions (LTC) Network, West Midlands Academic Health Science Network (WMAHSN), Birmingham; Clinical Lead, the Staffordshire STP's digital work stream's technology-enabled care services sub-programme. Stafford; and Honorary Professor, Keele University, Keele.

\section{Marc Schmid,}

Director, Redmoor Health social enterprise, Chorley; Director, Digital Health Academy CIC, Chorley.

\section{Funding}

Ruth Chambers undertook the work that fed into the thinking captured in this editorial in her Staffordshire STP's digital workstream role, her WMAHSN LTC Network role. So these were funded by Stoke-on-Trent and North Staffs CCGs (STP role) and WMAHSN. Marc Schmid fed in his thinking from experience working with Stokeon-Trent \& North Staffs CCGs' practices and local trust - funded by the CCGs and WMAHSN - as a digital champion with the LTC Network. We wrote the publication while undertaking our

\section{ADDRESS FOR CORRESPONDENCE}

\section{Ruth Chambers}

Stoke-on-Trent CCG, One Smithfield, Hanley, Stoke-on-Trent, ST1 4FA, UK.

\section{E-mail: ruth.chambersđastoke.nhs.uk}

roles. The Good Things Foundation (declared in Acknowledgements) pathfinder wave 2 has allotted €50k for the project - neither author has been paid anything from this fund yet or for this work.

\section{Provenance}

Commissioned; not externally peer reviewed.

\section{Acknowledgements}

The Good Things Foundation has awarded national wave 2 pathfinder status to Stoke-on-Trent CCG for digital upskilling of citizens with long-term conditions, where Ruth Chambers is the clinical lead. The content of the editorial captures learning focused on patients' needs and preferences for technology-enabled care.

\section{DOI: https://doi.org/10.3399/bjgp18X694877}

\section{REFERENCES}

1. Chambers R, Schmid M, Birch-Jones J. Digital healthcare: the essential guide. Oxford: Otmoor Publishing, 2016.

2. Health Education England, Royal College of Nursing. Improving digital literacy. London: RCN, 2017.

3. Marszal J, Donnelly A, Chambers R. Helping the elderly take tablets. Health 212016 ; 1 Nov: http://www.health21.org.uk/2016/11/01/ old-people-will-use-tech-if-clinicians-letthem/ laccessed 2 Feb 2018).

4. Staffordshire Digital Design Authority. Code of practice for technology enabled care services for Staffordshire Local Digital Roadmap. 2017. http://www.digitalhealthsot. nhs.uk/index.php/clinicians-learning-centre/ cpd-programme/cpd-certificate/cop-sldr laccessed 2 Feb 2018).

5. Cottrell E, McMillan K, Chambers R. A cross-sectional survey and service evaluation of simple telehealth in primary care: what do patients think? BMJ Open 2012; Jan: http://bmjopen.bmj.com/cgi/content/full/ bmjopen-2012-001392 (accessed 2 Feb 2017).

6. Collis L, Whitmore M, Chambers R Telehealth in primary care: what's in it for me? Practice Nursing 2014; 25(8): 406-408.

7. Moore K, Cottrell E, Chambers R. Facebook in general practice: a service evaluation in one health economy. BJGP Open 2017; DOI https://doi.org/10.3399/bjgpopen17X101181.

8. Meigh S. SoMe in cardiovascular care: like, comment and share. Br J Cardiac Nurs 2017; 12(12): 594-595. https://wnw. magonlinelibrary.com/doi/abs/10.12968/ bjca.2017.12.12.594 (accessed 2 Feb 2018).

9. Chambers R, Schmid M. How a Skype trolley saves GP time. Pulse 2016; 26 Sep: http://www.pulsetoday.co.uk/your-practice/ focus-on/how-a-skype-trolley-saves-gptime/20032834.article (accessed 2 Feb 2018). 